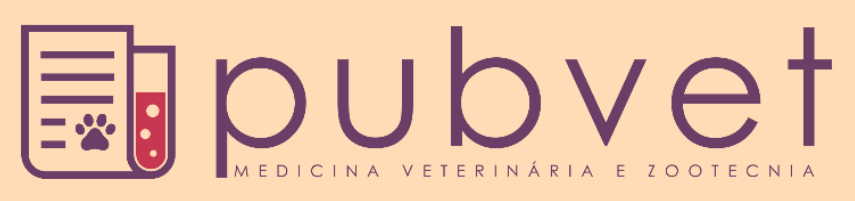

https://doi.org/10.31533/pubvet.v14n2a515.1-5

\title{
Estudo retrospectivo das intervenções fisioterapêuticas realizadas em um hospital veterinário universitário na região centro oeste
}

\author{
Kamylla Caroline Santos ${ }^{1 *}{ }^{\bullet}$, Andréia Vitor Couto do Amaral $^{2} \bullet$, Júlia de Miranda Morais ${ }^{3}$, \\ Benedito Matheus dos Santos $^{4}{ }^{\bullet}$, Maisa Ribeiro ${ }^{5}$, Nadiene Alves Martins ${ }^{1}$, Jéssica Bueno \\ Guimarães $^{6}$, Gustavo Henrique Marques Araújo ${ }^{2}{ }^{\circ}$ \\ IAluna do Programa de Pós-graduação em Biociência Animal. Universidade Federal de Goiás. Jataí - GO Brasil. \\ ${ }^{2}$ Professor(a) doutor(a) do Programa de Pós-graduação em Biociência Animal, Universidade Federal de Goiás. Jataí - GO Brasil. \\ ${ }^{3}$ Professora doutora do Departamento de Medicina da Universidade Federal de Goiás, Jataí- GO Brasil. \\ ${ }^{4}$ Aluno do Programa de Pós-graduação em Farmácia. Faculdade de Ciências Farmacêuticas, Universidade de São Paulo. SP Brasil. \\ ${ }^{5}$ Professor do curso de Biomedicina da Universidade Federal de Jataí. \\ ${ }^{6}$ Medica veterinária pela Universidade Federal de Goiás. Mestrado em Biociência animal pela Universidade Federal de Jataí. Brasil. \\ *Autor para correspondência, E-mail: kamyllacarolinefisio@gmail.com
}

Resumo. Avaliaram-se retrospectivamente as intervenções fisioterapêuticas realizadas em cães e gatos atendidos no Hospital veterinário da Faculdade de Medicina Veterinária da Universidade Federal de Goiás - Regional Jataí durante o período de 2018 a 2019. De um total de 10 intervenções com a instituição de protocolos fisioterapêuticos, 6 foram na espécie canina e 4 na espécie felina gatos. Ao considerar a idade dos pacientes atendidos, predominou os animais mais velhos. Porém com uma ampla variação, com idades entre cinco meses a oito anos. Observando-se as raças, notou-se que o número de animais sem raça definida (SRD) foi predominante no período acompanhado. Em relação às doenças diagnosticadas com maior frequência durante o período analisado as lesões cutâneas aparecem em primeiro lugar, seguida de disfunções motoras, em terceiro as lesões de membro torácico direito (MTD), e por fim lesões em membros pélvicos (MP). O conhecimento das alternativas e ou terapias complementares para prevenção, tratamento e reabilitação de patologias que acometem a funcionalidade dos animais são de estrema importância para aqueles que atuam na área, além de servir para evidenciar o uso da Fisioterapia em Hospitais Veterinários.

Palavras chave: atendimento fisioterapêutico, medicina veterinária, reabilitação animal

\section{Retrospective study of physiotherapeutic interventions performed at a veterinary university hospital in the Midwest region}

\footnotetext{
Abstract. We retrospectively evaluated the physical therapy interventions performed on dogs and cats treated at the Veterinary Hospital of the Faculty of Veterinary Medicine of the Federal University of Goiás - Regional Jataí from 2018 to 2019. From a total of 10 interventions with the establishment of physical therapy protocols, 6 were in canine species and 4 in feline cats. When considering the age of the patients treated, the older animals predominated. But with a wide range, ranging from five months to eight years. Observing the breeds, it was noted that the number of animals without defined breed (SRD) was predominant in the period followed. Regarding the diseases most frequently diagnosed during the analyzed period, the cutaneous lesions appear first, followed by motor dysfunctions, third the right thoracic limb (BAT) lesions, and finally the pelvic limb (MP) lesions. The knowledge of alternatives and or complementary therapies for prevention, treatment and rehabilitation of pathologies that affect the functionality of animals are
} 
extremely important for those who work in the area, besides serving to highlight the use of Physiotherapy in Veterinary Hospitals.

Keywords: physiotherapeutic care, veterinary medicine, animal rehabilitation

\title{
Estudio retrospectivo de intervenciones fisioterapéuticas realizadas en un hospital veterinario universitario en la región del Medio Oeste.
}

\begin{abstract}
Resumen. Evaluamos retrospectivamente las intervenciones de fisioterapia realizadas en perros y gatos tratados en el Hospital Veterinario de la Facultad de Medicina Veterinaria de la Universidad Federal de Goiás - Jataí Regional de 2018 a 2019. De un total de 10 intervenciones con el establecimiento de protocolos de fisioterapia, 6 eran en especies caninas y 4 en felinos. Al considerar la edad de los pacientes tratados, predominaron los animales de edad mas avanzada. Pero con una amplia gama, que van desde cinco meses a ocho años. Al observar las razas, se observó que el número de animales sin raza definida (SRD) era predominante en el período seguido. En cuanto a las enfermedades diagnosticadas con mayor frecuencia durante el período analizado, las lesiones cutáneas aparecen primero, seguidas de las disfunciones motoras, en tercer lugar, las lesiones de la extremidad torácica derecha (BAT) y finalmente las lesiones de la extremidad pélvica (MP). El conocimiento de alternativas y / o terapias complementarias para la prevención, tratamiento y rehabilitación de patologías que afectan la funcionalidad de los animales son extremadamente importantes para quienes trabajan en el área, además de servir para resaltar el uso de la fisioterapia en los hospitales veterinarios.
\end{abstract}

Palabras clave: cuidado fisioterapéutico, medicina veterinaria, rehabilitación animal

\section{Introdução}

A fisioterapia é uma ciência que estuda, diagnostica, previne e trata disfunções cinéticas funcionais de órgãos e sistemas do corpo humano (Kolb, 1984; Reece \& Penteado Júnior, 1996). Seu exercício acontece em todos os níveis de atenção à saúde, com extrema produtividade na atuação multiprofissional, interdisciplinar e transdisciplinar (Dukes et al., 2006; Engelking, 2010). É indicada no tratamento de animais que necessitam de programas apropriados e específicos de reabilitação, os quais seguem os mesmos princípios da fisioterapia humana, reunindo dados, avaliando a condição física do animal, bem como seu quadro ortopédico e neurológico (Levine et al., 2008).

A reabilitação na medicina veterinária segue em interface com a fisioterapia, podendo a aplicação de técnicas fisioterapêuticas em animais ser realizada apenas por médicos veterinários especializados, com amplos conhecimentos e condições de avaliar o animal em sua anatomia, fisiologia, biomecânica, patologia clínica e cirúrgica (Pedro \& Oliveira, 2009; Sguarizi, 2007). Fato este, que vem levantando conflitos, visto que, com o desenvolvimento dessa área graças a crescente procura dos tutores dos animais de estimação por tratamentos menos invasivos, com custo mais acessivo e boa eficácia, vários profissionais vem migrando para o ramo, muitas vezes sem formação e capacitação necessárias, o que se torna um problema latente da fisioterapia veterinária (Grognet, 2009).

A Fisioterapia animal faz uso de técnicas não invasivas utilizadas na reabilitação de lesões em animais; seu entendimento e acompanhamento são de fundamental importância na prática clínica. Sendo assim, este trabalho se objetiva apresentar um estudo retrospectivo das intervenções fisioterapêuticas realizadas em cães e gatos atendidos no Hospital veterinário da Faculdade de Medicina Veterinária da Universidade Federal de Goiás - Regional Jataí no período de 2018 a 2019.

\section{Material e métodos}

Foi realizado um estudo retrospectivo dos atendimentos que utilizavam protocolos fisioterapêuticas realizadas em cães e gatos atendidos no Hospital veterinário da Faculdade de Medicina Veterinária da Universidade Federal de Goiás - Regional Jataí no período de agosto de 2018 a agosto 2019. Realizouse uma pesquisa nos arquivos de laudos do serviço de Fisioterapia animal, considerando os dados 
disponibilizados nas fichas de evolução dos pacientes. Foi realizada uma análise descritiva do número de casos que necessitaram de intervenções fisioterapêuticas.

Os dados foram registrados em planilha eletrônica (Excell-Microsoft Office ${ }^{\circledR}$ ), o que permitiu a montagem de tabelas e gráficos. Os resultados obtidos foram expressos mediante a distribuição de frequências e respectiva análise descritiva.

Foram atendidos os aspectos éticos da pesquisa, citando todos os autores utilizados no estudo, respeitando a norma brasileira regulamentadora NBR-6023, que permite a identificação de elementos a serem incluídos e orienta a compilação e produção de referências. Os dados foram utilizados exclusivamente com finalidade científica.

\section{Resultado e discussão}

No período proposto, foram observados 10 casos com a instituição de protocolos fisioterapêuticos no Hospital Veterinário. Desses, seis foram na espécie canina e quatro na espécie felina (gatos), no setor de fisioterapia do Hospital veterinário da Faculdade de Medicina Veterinária da Universidade Federal de Goiás - Regional Jataí. Na tabela 1 também é possível verificar a distribuição de acordo com o sexo.

Tabela 1. Distribuição de pacientes atendidos no Serviço de Fisioterapia do Hospital Veterinário da Regional Jataí, da UFG, no período de agosto de 2018 a agosto de 2019, de acordo com o sexo e a espécie.

\begin{tabular}{lcc}
\hline Sexo & Felinos & Caninos \\
\hline Fêmeas & 2 & 4 \\
Machos & 2 & 2 \\
Total & 4 & 6 \\
\hline
\end{tabular}

De acordo com a tabela 2, ao considerar a idade dos pacientes atendidos, predominou os animais mais velhos. Entretanto, observou-se uma ampla variação, com idades entre cinco meses a oito anos, sendo que, os animais com menos de um ano constituíram a minoria. Pode-se justificar esse fato pelo estudo que mostra que com o envelhecimento, a necessidade de fisioterapia aumenta, em decorrência de doenças degenerativas (Alencar et al., 2017).

Tabela 2. Distribuição de pacientes atendidos no Serviço de Fisioterapia do Hospital Veterinário da Regional Jataí, da UFG, no período de agosto de 2018 a agosto de 2019 , de acordo com a idade.

\begin{tabular}{lc}
\hline Idade & Número de animais \\
\hline$<1$ ano & 1 \\
1 a 4 anos & 4 \\
5 a 8 anos & 5 \\
\hline
\end{tabular}

As raças atendidas observadas foram um Poodle, um São Bernardo, um Dachshund, três cachorros sem raça definida e quatro gatos sem raça definida. O número de animais sem raça definida (SRD) foi predominante no período acompanhado, provavelmente pela grande variedade de cruzamentos entre as raças, o que resulta em animais sem raça definida. Em relação às doenças diagnosticadas com maior frequência durante o período analisado as lesões cutâneas aparecem em primeiro lugar, seguida de disfunções motoras, em terceiro as lesões de membro torácico direito (MTD), e por fim lesões em membros pélvicos (MP) conforme representa a figura 1.

A fisioterapia animal utiliza-se de uma variedade de condutas que devem ser aplicadas de acordo com a avaliação minuciosa do paciente, considerando a origem, gravidade dos sinais clínicos a progressão da doença (aguda ou crônica) e a participação do animal e, especialmente, o envolvimento do proprietário em todas as fases da reabilitação. Objetivando preservar, manter e restaurar a integridade física e funcional, de forma a promover o bem-estar e a qualidade de vida do animal. Reduzindo sua dor, aumentando a funcionalidade, amplitude de movimento, mobilidade, fortalecimento muscular, além de diminuir o tempo de cura das lesões, corrigir problemas posturais, melhorar a qualidade da cicatrização e prevenir o desenvolvimento de contraturas e fibroses de tecidos moles, de forma a devolver ao paciente sua função no máximo nível da normalidade (Levine et al., 2008; Pedro \& Oliveira, 2009). 


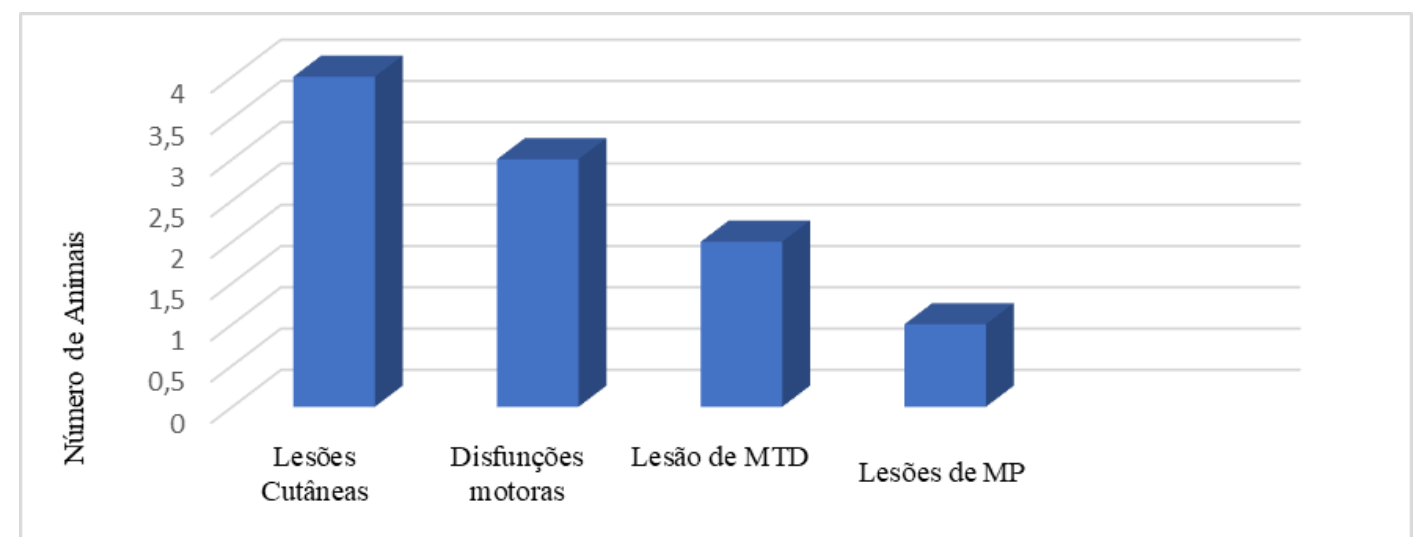

Figura 1. Distribuição de pacientes atendidos no Serviço de Fisioterapia do Hospital Veterinário da Regional Jataí, da UFG, no período de agosto de 2018 a agosto de 2019, de acordo com as patologias apresentadas.

Dentre as condutas fisioterapêuticas empregadas em animais, destacam-se a utilização de recursos eletrotermofototerapêuticos (TENS, interferencial, laser; ultrassom, ondas de choque, micro-ondas, ondas curtas), técnicas manuais (massoterapia, quiropraxia, osteopatia, mobilização, manipulação, mobilização neural), recursos termoterapêuticos físicos (crioterapia e calor), além da hidroterapia; dos alongamentos, da estabilização segmentar e funcional e da cinesioterapia (Alves et al., 2019; Nogueira et al., 2010).

Esses recursos empregados em conjunto com a terapêutica médica e cirúrgica, podem proporcionar uma recuperação mais rápida e completa do animal, independentemente do tratamento escolhido (conservativo ou cirúrgico) (Brisson, 2010; Grognet, 2009).

A terapia física veterinária (fisioterapia) segundo a American Veterinary Medical Association (AVMA, 2007) compreende o uso de técnicas não invasivas utilizadas na reabilitação de lesões em animais. Essa definição exclui grande parte da prática habitual da fisioterapia, como o diagnóstico fisioterapêutico e a prevenção de lesões, verificando assim uma discordância na exatidão do campo de atuação. Nesse sentido, uma abordagem multidisciplinar asseguraria ao animal o benefício dos melhores tratamentos permissíveis a sua saúde.

Embora haja um uso crescente das técnicas de reabilitação animal, há relativamente pouca literatura científica disponível. Apesar de ser uma área relativamente nova, a Fisioterapia veterinária encontra-se em grande expansão desde os últimos anos, mostrando bons resultados principalmente no tratamento de regeneração tecidual e disfunções motoras.

\section{Conclusão}

A fisioterapia se mostra como eficiente alternativa ou terapia complementar na prevenção, tratamento e reabilitação de diversas patologias que acometem a funcionalidade dos animais. O estudo retrospectivo apresentado possibilita evidenciar o uso da Fisioterapia no Hospital Veterinário da Universidade Federal de Goiás - Regional Jataí, em atendimentos a cães e gatos.

\section{Referências bibliográficas}

Alencar, M. d. C. B., Henemann, L. \& Rothenbuhler, R. (2017). A capacidade funcional de pacientes, e a fisioterapia em um programa de assistência domiciliar. Fisioterapia em Movimento, 21(1).

Alves, M. V. d. L. D., Sturion, M. A. T. \& Gobetti, S. T. C. (2019). Aspectos gerais da fisioterapia e reabilitação na medicina veterinária. Ciência Veterinária UniFil, 1(3):69-78.

Brisson, B. A. (2010). Intervertebral disc disease in dogs. The Veterinary Clinics of North America. Small Animal Practice, 40(5):829-858.

Dukes, H. H., Reece, W. O., Figueiredo, C., Vanzellotti, I. R. \& Zanon, R. F. (2006). Fisiologia dos animais domésticos (Vol. 1): Guanabara Koogan.

Engelking, L. (2010). Fisiologia endócrina e metabólica em medicina veterinária. São Paulo, Brasil: Roca. 
Grognet, J. (2009). Animal physiotherapy - assessment, treatment and rehabilitation of animals. The Canadian Veterinary Journal, 50(3):1-7.

Kolb, E. (1984). Fisiologia veterinária. Rio de Janeiro: Koogan.

Levine, D., Millis, D. L., Marcellin-Little, D. J. \& Taylor, R. (2008). Reabilitação e fisioterapia na prática de pequenos animais. São Paulo, Brasil: Roca.

Nogueira, J. L., Silva, M. V. M., Araíjo, K. P. C. \& Ambrósio, C. E. (2010). A utilização da hidroterapia como um recurso da fisioterapia veterinária. Revista Eletrônica de Medicina Veterinária, 141-7.

Pedro, C. R. \& Oliveira, S. P. (2009). Curso de Fisioterapia Veterinária. Instituto Brasileiro de Reabilitação Animal. ANFIVET - Associação Nacional de Fisioterapia Veterinária, 17-10.

Reece, W. O. \& Penteado Júnior, N. (1996). Fisiologia de animais domésticos. São Paulo: Roca.

Sguarizi, G. (2007). CFMV regulamenta fisioterapia veterinária. Revista Conselho Regional de Medicina Veterinária-PR, 2210-11.

Willis, N.G., Monroe, F.M., Potworowski, J.A; Halbert, G., Evans, B.R; Smith, J.E., Andrews, K.J; Spring, L. \& Envisioning, A. B. (2007)The future of veterinary medical education: the Association of American Veterinary Medical Colleges Foresight Project, final report. Journal of Veterinary Medical Education, 34(1), 1-41.

Recebido: 4 de janeiro, 2020.

Aprovado: 1 de fevereiro, 2020.

Publicado: 21 de março, 2020.

Licenciamento: Este artigo é publicado na modalidade Acesso Aberto sob a licença Creative Commons Atribuição 4.0 (CC-BY 4.0), a qual permite uso irrestrito, distribuição, reprodução em qualquer meio, desde que o autor e a fonte sejam devidamente creditados 